\title{
Criminologie
}

\section{La communauté thérapeutique du pénitencier de Donnacona : un an déjà !}

\section{Lise Turcotte}

Volume 22, numéro 1, 1989

Drogues et criminalités

URI : https://id.erudit.org/iderudit/017276ar

DOI : https://doi.org/10.7202/017276ar

Aller au sommaire du numéro

\section{Éditeur(s)}

Les Presses de l'Université de Montréal

ISSN

0316-0041 (imprimé)

1492-1367 (numérique)

Découvrir la revue

Citer cet article

Turcotte, L. (1989). La communauté thérapeutique du pénitencier de Donnacona : un an déjà ! Criminologie, 22(1), 111-119.

https://doi.org/10.7202/017276ar
Résumé de l'article

It was in 1987 that the program for the treatment of drug addiction was started at the Donnacona Penitentiary, based on the Therapeutic Community model. It was one of the regional initiatives taken under the National Anti-Drug campaign launched in the spring of 1987. This program is to be the major regional undertaking in Quebec in the field of antidrug programs in the prison milieu.

In this article, we discuss the program as it is applied at Donnacona and the persons who work there as counsellors.

We then draw a general criminological profile of the inmates that are following the program and the results obtained.

Finally, we mention some of the strong points of the program and some of its limitations or difficulties in applying this model in the prison milieu. 


\section{Témoignage}

\section{LA COMMUNAUTE THÉRAPEUTIQUE DU}

PÉNITENCIER DONNACONA : UN AN DÉJÀ!

Lise Turcotte*

It was in 1987 that the program for the treatment of drug addiction was started at the Donnacona Penitentiary, based on the Therapeutic Community model. It was one of the regional initiatives taken under the National Anti-Drug campaign launched in the spring of 1987. This program is to be the major regional undertaking in Quebec in the field of antidrug programs in the prison milieu.

In this article, we discuss the program as it is applied at Donnacona and the persons who work there as counsellors.

We then draw a general criminological profile of the inmates that are following the program and the results obtained.

Finally, we mention some of the strong points of the program and some of its limitations or difficulties in applying this model in the prison milieu.

C'est en octobre 1987 que débutait le programme de traitement pour toxicomanes de l'Établissement Donnacona basé sur le modèle de la Communauté thérapeutique. Il s'agissait d'une des initiatives régionales prises dans le cadre de la Stratégie Nationale Antidrogue élaborée au printemps 1987. Ce programme se veut l'initiative majeure de la région du Québec dans le domaine des programmes antidrogues en milieu carcéral.

Dans ce texte, nous discuterons du programme tel qu'il est appliqué à Donnacona et des personnes qui y travaillent comme intervenants, c'est-à-dire les agents-conseillers.

Nous tracerons, par la suite, les grandes lignes du profil criminologique des détenus qui suivent ce programme et des résultats obtenus.

Finalement, nous mentionnerons quelques points forts et certaines limites ou difficultés d'application de ce modèle en milieu carcéral.

\section{CE PROGRAMME SE DISTINGUE DE PLUSIEURS FAÇONS :}

- Il est une première au Canada au sens où il se situe dans un établissement carcéral fédéral.

- Il se déroule dans une unité complètement fermée.

* Gérante de l'unité pour toxicomanes du pénitencier de Donnacona, Établissement Donnacona, 1538 route 138 , Donnacona, Québec, G0A 1T0. 
- Son modèle d'intervention repose sur la Communauté thérapeutique.

- Les interventions cliniques sont effectuées par des officiers de correction spécialement formés.

\section{LE PROGRAMME}

Le programme comme tel se divise en deux temps:

ler temps : traitement extensif de 9 à 12 mois à l'unité Donnacona;

2e temps: phase de réinsertion sociale d'une durée de 4 mois en Libération conditionnelle de jour (L.C.J.) ou totale au Centre de jour de Portage, rue Ste-Famille, Montréal.

\section{ler TEMPS : TRAITEMENT INTENSIF À DONNACONA}

\section{PHASE I : PHASE D'INDUCTION AU PROGRAMME}

Cette phase d'une durée de 21 jours est une variante du modèle de Portage. Elle s'est avérée nécessaire pour au moins trois raisons:

- elle protège la Famille ou la Communauté (les résidents déjà impliqués dans le traitement) contre l'intrusion trop rapide d'un nouveau membre qui fonctionnerait encore selon la loi du milieu et qui tenterait invariablement de continuer à l'appliquer;

- elle permet au personnel d'observer le candidat et d'envisager un retrait du programme si nécessaire;

- elle permet au candidat de se familiariser avec le langage et les outils thérapeutiques utilisés dans la communauté.

Dès le début de cette phase, le nouveau membre est invité à écrire l'histoire de sa vie. Les agents conseillers le rencontrent pour l'informer sur le fonctionnement général de la Communauté thérapeutique et une documentation pertinente lui est remise.

Le sixième jour, il est rencontré en entrevue d'admission. Cette entrevue, d'une durée d'une heure environ, sert à évaluer la motivation du sujet et surtout ses capacités personnelles et psychologiques à bénéficier du traitement.

Si le candidat réussit cette épreuve, il est présenté à la communauté et invité à signer son «engagement personnel» qui le lie au programme et à ses règlements pour la durée de son séjour à l'unité. 
Si le candidat échoue, il quitte l'unité et son retour à l'établissement d'origine est organisé suivant la procédure habituelle de transfert. Parfois, la problématique du sujet fait que l'entrevue peut se faire en deux étapes pendant la phase d'induction.

Jusqu'ici, la majorité des candidats acceptés à l'unité ont réussi l'entrevue d'accueil.

Pendant les deux dernières semaines d'induction, le candidat s'initie, avec les autres nouveaux et les agents conseillers, aux différents outils thérapeutiques.

\section{PHASE II : LE TRAITEMENT ET SES ÉTAPES}

À la fin de la phase d'induction, soit le $21 \mathrm{e}$ jour, le candidat intègre la Famille comme nouveau membre.

À partir de ce moment, le nouveau membre est pris en charge par la Famille, c'est-à-dire les pairs sur qui repose la responsabilité de l'aider à régler son problème de toxicomanie.

Pour respecter le modèle de la Communauté thérapeutique, les agents conseillers interviennent maintenant en troisième lieu, après l'intervention des membres intermédiaires et des vieux membres.

La Famille a la responsabilité de créer un climat thérapeutique exigeant, forçant les modifications d'attitudes et permettant finalement des changements en profondeur dans la dynamique de la personnalité du résident.

Tout d'abord, chacun devient le miroir de l'autre et les mécanismes de défense qui ont contribué à construire l'image du délinquant doivent être systématiquement dénoncés pour que l'intervention puisse rejoindre le «toxicomane».

Ce dernier est par la suite amené à entrer de plus en plus en contact avec sa souffrance, ses peurs et ses lacunes. Il doit d'abord identifier le pourquoi de sa consommation de drogue pour parvenir à une prise de conscience de la blessure narcissique sous-jacente.

Dans cette recherche de lui-même, il sera continuellement aidé par la Famille qui devient à la fois le catalyseur de cette remise en question et le support affectif essentiel pour y parvenir.

Concrètement, chacun doit prendre sa place dans le cercle familial et doit laisser à l'autre celle qui lui revient. Cependant, cet objectif ne 
peut être atteint sans que l'individu ne devienne un être responsable, honnête, franc, généreux et respectueux des autres... autant de défis à relever!

L'image très négative de lui-même, la passivité, l'égocentrisme, la dépendance, l'immaturité, sont autant de traits de personnalité que le toxicomane aura à modifier pendant sa thérapie pour parvenir, finalement, à un niveau de confiance et d'estime de soi suffisant pour lui permettre d'évoluer de façon adaptée dans la société.

\section{2e TEMPS : LA RÉINSERTION SOCIALE}

Lorsque le résident aura traversé toutes les phases du programme et atteint tous les objectifs fixés, il sera prêt à accéder à la réinsertion sociale, c'est-à-dire au deuxième temps du programme.

Elle est la plus critique socialement et émotionnellement car l'encadrement devient de moins en moins rigide. Elle permet de prendre une distance psychologique et émotive vis-à-vis de la communauté, à couper le cordon, à briser la dépendance à la Famille. Elle est un temps où les apprentissages de vie en société doivent être expérimentés et réussis.

\section{QUI SONT LES INTERVENANTS DANS CE PROGRAMME?}

D'abord la sélection des officiers de correction, qui agissent comme intervenants dans le programme, s'est faite sur une base volontaire parmi le personnel de Donnacona.

Les critères de sélection étaient les suivants:

- années d'expérience au Service correctionnel canadien (SCC);

- formation académique;

- assiduité au travail;

- profil de personnalité (c'est-à-dire vécu personnel et capacité à travailler en équipe).

\section{LA FORMATION DES INTERVENANTS}

Avant le début du premier programme, toute l'équipe s'est rendue au Centre Portage, Lac Écho, pour une formation d'une durée de dix jours. 
Pendant ces dix jours, l'équipe a expérimenté le modèle de la Communauté thérapeutique en se l'appliquant à elle-même sous la direction des intervenants de Portage.

Par la suite, toute cette valeureuse équipe est revenue à Donnacona accompagnée d'un membre de l'équipe de Portage qui a poursuivi la formation pendant trois mois sur une base intensive. Finalement, la supervision faite par Portage s'est poursuivie pendant les neuf mois suivants à raison d'une journée par semaine environ.

\section{L'ÉQUIPE PRÉSENTEMENT EN FONCTION}

L'équipe présente est composée d'officiers qui ont entre 2 et 12 ans d'expérience au S.C.C. comme officiers de correction :

- ils démontrent beaucoup d'intérêt dans leur travail;

- ils se sentent très valorisés;

- ils font preuve d'une très grande stabilité et

- l'absentéisme est très bas en comparaison avec les statistiques de l'ensemble du pénitencier.

À cette équipe s'ajoute une agente de gestion de cas et une psychologue.

Parmi les caractéristiques de l' unité, nous avons mentionné «unité fermée».

Pourquoi une unité fermée?

- D'abord l'unité est située dans un établissement à sécurité maximale afin d'accueillir les détenus provenant de trois niveaux sécuritaires : minimal, moyen et maximal.

- Il n'y a aucun contact physique et même visuel entre les détenus de la population régulière du pénitencier et ceux de l'unité toxicomanie.

- L'environnement sécurisant de l'unité permet au résident de laisser tomber peu à peu son image de détenu sans quoi toute intervention thérapeutique est constamment mise en échec.

- Les risques de trafic ou de consommation de drogues doivent, selon notre modèle, être réduits au strict minimum sinon nuls pendant le traitement. Le but visé étant de court-circuiter tous les mécanismes de défense, fermer toutes les portes y compris celle de la fuite dans la drogue pour amener le détenu à faire face à sa réalité. 


\section{BUT DU TRAITEMENT}

Les buts du traitement se décrivent comme suit :

- amener les résidents à devenir des invididus responsables;

- les aider à consolider leur identité et

- modifier ou leur donner un schème de valeurs acceptables socialement.

L'hypothèse de départ est que la toxicomanie est un symptôme et qu'à travers sa consommation de drogue/alcool, l'individu se fuit luimême et fuit sa réalité.

\section{MODĖLE D'INTERVENTION UTILISÉ À DONNACONA}

Comme nous l'avons mentionné préalablement, le modèle d'intervention utilisé à l'unité pour toxicomanes du pénitencier de Donnacona est celui de la Communauté thérapeutique. Il s'agit donc d'une thérapie intensive qui s'étale de $7 \mathrm{~h}$ à $21 \mathrm{~h} 30,7$ jours par semaine. Les expériences de vie quotidiennes et passées servent sans cesse d'éléments de confrontation et de remise en question.

C'est un milieu surprotecteur, et c'est voulu ainsi, pour amener le résident à se centrer uniquement sur lui-même et sur ses co-détenus qui constituent la Communauté. L'élément-clé qui rend ce modèle si efficace, en particulier avec une clientèle carcérale, est qu'il génère son propre traitement: les résidents traités se devant d'être en même temps les thérapeutes de leurs pairs.

\section{LES ACTIVITÉS DANS L'UNITÉ}

L'unité base ses interventions, en totalité, sur des activités de groupe: groupes de thérapie visant l'apprentissage de l'expression d'émotions telles la colère, la frustration, la peine, etc., plutôt que de les manifester dans l'«action-out» délictuel.

Les objectifs visés sont variés et tiennent compte des besoins de chacun, par exemple : tendre à devenir un être responsable; tenir compte des autres, de l'entourage, au lieu d'agir en fonction de soi-même et de la satisfaction immédiate de ses besoins; faire l'apprentissage des délais; se donner des objectifs réalisables; apprendre à se faire confiance et à faire confiance aux autres, etc. 
Une des hypothèses à la base de cette approche est qu'en rehaussant son estime de soi, l'individu aura moins ou pas besoin de recourir à la drogue pour se sentir quelqu'un.

La thérapie est continuelle dans les groupes de l'unité : pendant les repas, les pauses cigarettes, les travaux d'entretien, les marches dans la cour extérieure, les sports; tout peut être sujet à la confrontation ou/et à la remise en question.

Parmi les activités autres que les groupes de thérapie, il y a les sports, les arts plastiques, la relaxation, l'école, la messe du dimanche, les visites des parents et amis et les absences temporaires avec escorte pendant les derniers mois du programme pour préparer à la phase de réinsertion sociale.

\section{CONDITIONS D'ADMISSION}

Voici les conditions exigées à l'admission :

- être toxicomane ou avoir un problème sérieux de consommation de drogue;

- être éligible à une libération conditionnelle dans 9 à 12 mois avec de fortes chances de l'obtenir;

- être motivé à changer et

- posséder des ressources personnelles et intellectuelles suffisantes pour suivre le programme.

\section{QUELLE CLIENTÈLE CARCÉRALE CE PROGRAMME REJOINT-IL?}

Dans un premier temps, nous avons été forcés de constater encore une fois ce que nous savions déjà, c'est-à-dire que seulement une minorité de la population carcérale est prête à faire un effort pour s'en sortir.

Au début du programme, nous n'avons reçu que peu de demandes d'admission et beaucoup de détenus se désistaient après l'entrevue de sélection ou en début de programme. Un tiers des détenus abandonnent dans les trois premiers mois du programme parce qu'ils considèrent la thérapie trop difficile.

Jusqu'à maintenant (octobre 1988), trente-cinq candidatures ont été acceptées, vingt et un détenus participent présentement au programme et quatre autres s'y intégreront au début de novembre 1988. 
Les détenus viennent majoritairement d'établissements de niveau sécuritaire maximal. La moyenne des sentences est de huit ans. Ce sont pour la plupart des multirécidivistes, saturés de «faire du temps», conscients que la récidive est inévitable s'ils ne font pas un ultime effort pour changer quelque chose dans leur vie, avant leur libération.

\section{LES RÉSULTATS OBTENUS JUSQU'ICI}

En date du mois d'octobre 1988, trois résidents ont complété avec succès le programme de Donnacona et la libération conditionnelle leur a été octroyée. Quatre autres résidents devraient quitter le programme en janvier 1989.

\section{PHASE DE RÉINSERTION SOCIALE}

Ceux qui ont réussi le programme se rendent au centre Portage, rue Ste-Famille, à Montréal, pour la deuxième étape: celle de la réinsertion sociale. Cette réinsertion dure quatre mois.

Les individus reprennent alors contact avec la société, encadrés par les intervenants de Portage. Par la suite, ce sont les contacts avec le milieu familial et/ou certaines personnes importantes, tout en se ressourçant continuellement auprès du groupe de pairs à Portage. Puis viennent les apprentissages de recherche d'emploi, de logement; d'établissement, de budgets, de gestion du temps, etc. La porte vers la liberté s'entrouvre toujours davantage, mais Portage reste la ressource vers laquelle ils peuvent se tourner aussi longtemps qu'ils le désirent.

\section{POINTS FORTS DU MODĖLE D'INTERVENTION}

Le modèle de la Communauté thérapeutique permet de multiplier l'impact d'une intervention d'un participant par le nombre de participants; plus la communauté est nombreuse, plus elle est dynamique. L'intervention est d'autant plus sentie et pertinente qu'elle est faite par un autre toxicomane, le rôle des intervenants se limitant à encadrer cette intervention.

À ce stade-ci, les résultats démontrent que le choix du modèle était approprié à une clientèle carcérale toxicomane parce que ce modèle permet de déjouer finalement toutes manipulations, toutes rationalisations ou tous autres mécanismes de défense derrière lesquels l'individu s'est toujours caché. 


\section{LIMITES OU DIFFICULTÉS D'APPLICATION DU MODÈLE EN MILIEU CARCÉRAL}

Le programme s'adresse à une clientèle fortement criminalisée, d'où l'obligation de percer la structure criminelle avant de rejoindre le toxicomane lui-même.

La «loi du milieu» carcéral oblige à une sélection très serrée de la clientèle, sinon il y a risque d'éclatement de la communauté à l'arrivée d'un nouveau.

Les exigences de la Commission nationale des libérations conditionnelles (CNLC), que la libération conditionnelle soit accordée ou non, sont autant de contraintes avec lesquelles il faut composer tout en respectant les objectifs du programme.

En conclusion, nous considérons que les objectifs prévus à la fin de la première année du programme ont été atteints et que l'avenir est prometteur. 